Article

\title{
Cocirculation of Swine H1N1 Influenza A Virus Lineages in Germany
}

\author{
Roland Zell 1,*(D), Marco Groth ${ }^{2}$, , Andi Krumbholz ${ }^{1,3}$, Jeannette Lange ${ }^{1,4}$, Anja Philipps 1,5 \\ and Ralf Dürrwald 1,6 \\ 1 Section of Experimental Virology, Institute for Medical Microbiology, Jena University Hospital, Friedrich \\ Schiller University Jena, D-07745 Jena, Germany; krumbholz@infmed.uni-kiel.de (A.K.); \\ jeannette.lange@pei.de (J.L.); anja.philipps@thermofisher.com (A.P.); duerrwaldr@rki.de (R.D.) \\ 2 CF DNA Sequencing, Leibniz Institute on Aging, Fritz Lipmann Institute, D-07745 Jena, Germany; \\ marco.groth@leibniz-fli.de \\ 3 Institute of Infection Medicine, Kiel University and University Medical Center Schleswig-Holstein, \\ D-24105 Kiel, Germany \\ 4 Paul-Ehrlich-Institut, D-63225 Langen, Germany \\ 5 Thermo Fisher Scientific GENEART GmbH, D-93059 Regensburg, Germany \\ 6 Robert Koch Institute, D-13353 Berlin, Germany \\ * Correspondence: roland.zell@med.uni-jena.de
}

Received: 2 June 2020; Accepted: 14 July 2020; Published: 15 July 2020

\begin{abstract}
The genome analysis of $328 \mathrm{H} 1 \mathrm{~N} 1$ swine influenza virus isolates collected in a 13-year long-term swine influenza surveillance in Germany is reported. Viral genomes were sequenced with the Illumina next-generation sequencing technique and conventional Sanger methods. Phylogenetic analyses were conducted with Bayesian tree inference. The results indicate continued prevalence of Eurasian avian swine H1N1 but also emergence of a novel H1N1 reassortant, named Schneiderkrug/2013-like swine H1N1, with human-like hemagglutinin and avian-like neuraminidase and internal genes. Additionally, the evolution of an antigenic drift variant of A (H1N1) pdm09 was observed, named Wachtum/2014-like swine H1N1. Both variants were first isolated in northwest Germany, spread to neighboring German states and reached greater proportions of the H1N1 isolates of 2014 and 2015. The upsurge of Wachtum/2014-like swine H1N1 is of interest as this is the first documented persistent swine-to-swine spread of A (H1N1) pdm09 in Germany associated with antigenic variation. Present enzootic swine influenza viruses in Germany now include two or more co-circulating, antigenically variant viruses of each of the subtypes, H1N1 and H1N2.
\end{abstract}

Keywords: swine influenza virus; reassortment; H1N1; antigenic drift; phylogenetic analysis; Germany

\section{Introduction}

Influenza A viruses (IAV) of the family Orthomyxoviridae infect a wide range of hosts including feral water fowl (main hosts), poultry and several mammalian species (e.g., humans, pigs and horses). Swine influenza is of particular significance due to its great economic importance. Globally, there are three areas with major swine production, each with characteristic, enzootic swine influenza A virus (S-IAV) strains. These areas are Europe, North America and East/Southeast Asia. Furthermore, pig holding is increasing in Central and South America which probably also contributed to the emergence of A (H1N1) pdm09 [1].

Eurasian avian-like swine H1N1 (EA swH1N1) emerged in Belgium and Germany in 1979 [2,3] after transspecies infection of pigs with an avian IAV. This virus achieved sustained circulation in pigs and spread to many European countries but also to China and Korea [4,5]. Analysis of German avian IAV isolates which had been collected in the 1970s suggested that EA swH1N1 is a 
triple reassortant with a unique internal gene cassette (IGC) that had not been detected in avian IAVs before [6]. Later, EA swH1N1 reassorted with seasonal H3N2 and seasonal H1N1 to yield so-called human-like (hu-like) swH3N2 and hu-like swH1N2 (reviewed in [4]). Recent investigations of European S-IAV isolates revealed different lineages of swH1N2 and swH3N2 reassortants on the continent and the UK [7,8]. All lineages have the EA swH1N1 internal gene cassette (EA IGC), but hemagglutinin (HA) and neuraminidase (NA) genes are either A/Port Chalmers/1/1973-like (H3N2), A/Albany/20/1974-like (H3N2) or Singapore/6/1986-like (H1N1). Another independent hu-like swH1N2 reassortant came up in Italy [9]. This $7+1$ reassortant has an A/Singapore/6/1986-like (H1N1) HA gene, an A/Panama/2007/1999-like (H3N2) NA gene and the EA IGC. After the emergence of A (H1N1) pdm2009 ( $\left.\mathrm{H}_{\mathrm{pdm}} \mathrm{N} 1_{\mathrm{pdm}}\right)$, numerous anthroponotic infections of pigs have been detected in many European countries both at the ongoing pandemic and thereafter [7,10-14]. Due to significant cross-reaction of antibodies directed against EA swH1N1 [15], $\mathrm{H} 1_{\text {pdm }} \mathrm{N} 1_{\text {pdm }}$ failed to establish stable infection chains in pigs in Germany and other countries with high pig density and prevalence of EA swH1N1. However, numerous reassortants arose of which the Papenburg/2010-like swH1N2 viruses, a stable $7+1$ swH1N2 reassortant with $\mathrm{HA}$ and $\mathrm{IGC}$ of $\mathrm{H} 1_{\mathrm{pdm}} \mathrm{N} 1_{\mathrm{pdm}}$ and $\mathrm{NA}$ gene of the contintental Gent/1984-like swH3N2, became endemic in Germany, Belgium and the Netherlands [7,8,13,16].

Losses caused by swine influenza are economically significant. Therefore, swine influenza surveillance is of increasing importance. Recently, the ESNIP3 consortium reported results on a S-IAV survey conducted 2010-2013 in 14 European countries [17]. Independently, members of the German FluResearchNet consortium have conducted a 13-year long-term swine influenza surveillance in Germany, 2003-2015, which enabled the isolation of 1310 S-IAV isolates. The sequencing results of 267 swH1N2 isolates have been published recently [8]. The data indicated (i) the replacement of the continental Gent/1999-like swH1N2 by the Diepholz/2008-like, Emmelsbuell/2009-like and Papenburg/2010-like swH1N2 reassortants, and (ii) the emergence of other swH1N2 reassortants that were partly persistent (Gladau/2012-like swH1N2), partly ephemeric.

Here, we report on the genome analysis of 328 swH1N1 isolates, mostly from Germany. The present results demonstrate continued prevalence of EA swH1N1 but co-circulation with:

- $\quad$ A H1 $1_{\text {pdm }} \mathrm{N} 1_{\text {pdm }}$ variant which emerged in 2014 and lacks cross-reactivity with EA swH1N1 and $\mathrm{H} 1_{\text {pdm }} \mathrm{N} 1_{\text {pdm }}$, and

- A novel swH1N1 reassortant with the HA gene of the new prevalent Diepholz/2008-like swH1N2 lineage.

\section{Materials and Methods}

\subsection{Study Design}

The study design has been described previously (for details see [8]). Briefly, for a passive swine influenza survey, 8122 samples (7798 nasal swabs, 165 bronchoalveolar lavages, 159 lung tissues) of diseased pigs were sent in by veterinarians from 14 of the 16 German states. A few additional samples were from Austria, Switzerland and the Netherlands. All samples were analyzed by S-IAV-specific RT-PCR and virus isolation. Of 1310 virus isolates 810 were selected for sequencing, 327 of which were of the H1N1 subtype. In addition, $21 \mathrm{H1N1}$ isolates collected between 1979 and 2001 were included in the molecular analysis, of which the genomes of 16 had been published previously [6,13,18]. All in all, 348 H1N1 S-IAV genomes were analyzed, 328 of which are new S-IAV genomes. Supplementary Table S1 summarizes GenBank accession numbers, country and sampling dates of all S-IAV H1N1 strains sequenced by the Jena Swine Influenza Virus Sequencing Initiative (SIVSI).

\subsection{Cell Lines, Virus Isolation and Virus Amplification}

Madin-Darby bovine kidney cells (MDBK, ATCC CCL-22) and Madin-Darby canine kidney cells (MDCK, ATCC CCL-34) were maintained in Dulbecco's modified MEM (DMEM) supplemented with $10 \%$ fetal bovine serum, $100 \mathrm{U} / \mathrm{mL}$ penicillin and $100 \mu \mathrm{g} / \mathrm{mL}$ streptomycin. For virus isolation, 
PCR-positive samples were used for inoculation of embryonated hen eggs and MDBK cells. All virus isolates were typed by hemagglutination inhibition assays and RT-PCR as described in [17,19]. Virus was propagated in MDCK/MDBK cells using serum-free DMEM with $3 \mu \mathrm{g} / \mathrm{mL}$ trypsin and $25 \mathrm{mM} \mathrm{MgCl}_{2}$; cells were infected at a multiplicity of infection of 0.01 . After 2-3 days, supernatants were centrifuged at $1000 \times g$ and aliquoted. Virus stocks were stored at $-80{ }^{\circ} \mathrm{C}$ until use.

\subsection{Nucleic Acid Extraction and PCR Analysis}

For RNA extraction following kits were used: QIAamp Viral RNA Mini Kit (Qiagen, Hilden, Germany) and Chemagic Viral DNA/RNA Kit (PerkinElmer, Waltham, MS, USA). For diagnostics, reverse transcription was performed with the Superscript One step RT-PCR kit (Invitrogen). The following oligonucleotide primers were used for PCR diagnostics: M: FLU-M52F: 5'-CTTCTAACCGAGGTCGAAACG-3', FLU-M253R: $\quad$ 5'-AGGGCATTTTGGACAAAKCGTCTA-3' [19]; H1 (H1N1): H1-550F: 5'-AACAAYAARGRGAAAGAAGT-3' H1-1016R: 5'-GGGACDTTYCTTARTCCTGT-3'; H1 (H1N2): 18F: 5'-AACAATAGAGAAGAAGAAGT-3'， 7R: 5'-GGAATGTTCCTTAGTCCTGT-3' ; panH1N1: $\quad$ pH1-F238: 5'-GGAAATCCAGAGTGTGAATCACTC-3', pH1-R463: 5'-GAGGACATGCTGCCGTTACACC-3'; N1: NAN-1F: 5'-CGATGGACCAAGTAATGGGC-3' , NAN-1R: $5^{\prime}$-AATGGCAACTCAGCACCGTC-3'; conditions: $30 \mathrm{~min}$ at $45^{\circ} \mathrm{C}(1 \times), 15 \mathrm{~min}$ at $95^{\circ} \mathrm{C}(1 \times)$, $0.5 \mathrm{~min}$ at $94^{\circ} \mathrm{C}, 0.5 \mathrm{~min}$ at $48^{\circ} \mathrm{C}, 1 \mathrm{~min}$ at $68^{\circ} \mathrm{C}(35 \times), 10 \mathrm{~min}$ at $68^{\circ} \mathrm{C}(1 \times), 0.016 \mathrm{~min}$ at $20{ }^{\circ} \mathrm{C}(1 \times)$, thereafter at $4^{\circ} \mathrm{C}$.

\subsection{Sequence Analysis}

Plaque-purified swH1N1 isolates were used for RNA extraction and subsequent conventional Sanger sequencing or for Illumina sequencing as described previously [13,20]. Total RNA was prepared employing the Qiagen RNeasy Mini Kit (Qiagen, Hilden, Germany). For cDNA synthesis, $5 \mu \mathrm{g}$ of total RNA were reverse transcribed utilizing the universal influenza virus primer (5'-RGCRAAAGCAGG-3') [21] and $20 \mu \mathrm{L}$ RevertAid premium reverse transcriptase solution (ThermoFisher Scientific, St. Leon-Rot, Germany) following the manufacturer's protocol. Then, segment-specific amplification was conducted with published oligonucleotide primers [22]. PCR products were purified and sequenced employing the CEQ DTCS Quick Start Kit (Beckman Coulter, Krefeld, Germany).

Methods of Illumina sequencing of IAV and de novo assembly of virus genomes has been described in [13], except that sequencing was run on another platform (GenomeAnalyzer IIx or HiSeq2000/2500). Consensus sequences were determined by mapping the reads to reference genomes as described in [23]. Assignment to IAV lineages was based on the assembled contigs. All sequences were submitted to GenBank (accession numbers MK364805-MK367387; compare Supplementary Table S1).

\subsection{Phylogenetic Analyses}

Only complete open reading frames (HA-ORF: $1707 \mathrm{nt}$; NA-ORF: $1413 \mathrm{nt}$; PB2-ORF: $2280 \mathrm{nt}$; PB1-ORF: 2274 nt; PA-ORF: 2151 nt; NP-ORF: 1497 nt; M1/M2-encoding gene region: 982 nt; NS1/NS2-encoding gene region: $835 \mathrm{nt}$ ) were used for phylogenetic analyses. Additional sequences were retrieved from public databases (GenBank, GISAID) and aligned with MEGA5.2 [24]. For alignments, $950 \mathrm{HAH} 1$ sequences, 577 NAN1 sequences and $258 \mathrm{HAH} 1_{\mathrm{pdm}}$ sequences were compiled depending on the availability of sequence data. Two coalescent tree inference methods (MrBayes, BEAST) were used for phylogenetic analyses [25,26] applying optimal substitution models on the basis of the Bayesian information criterion and the corrected Akaike information criterion. The substitution model was selected with the find-best-model option implemented in MEGA5.2 and Mega X [24,27]. 


\subsection{Nomenclature of S-IAV Lineages and Clades}

Designations of S-IAV lineages which are established in literature like European/Eurasian avian (EA) swH1N1, human-like (hu-like) swH1N2 and hu-like swH3N2 were retained. For subgrouping of the HAH1 gene, the lineage and sublineage designations of Anderson et al. [28] were adopted (hereafter referred to as Anderson-2016 nomenclature). Reassortant Diepholz/2008-like, Emmelsbuell/2009-like, Papenburg/2010-like, Gladau/2012-like swH1N2 viruses have been introduced recently [8]. The novel viruses described here were named after a representative isolate, Schneiderkrug/2013-like swH1N1 and Wachtum/2014-like swH1 $1_{\mathrm{pdm}} \mathrm{N} 1_{\mathrm{pdm}}$. The prefix sw (swine) indicates the porcine host. Seasonal human H1N1 and A(H1N1)pdm2009 virus were distinguished by adding the subscripts 'seas' and 'pdm' to type designations $\left(\mathrm{H} 1_{\text {seas }} \mathrm{N} 1_{\text {seas }}, \mathrm{H} 1_{\text {pdm }} \mathrm{N} 1_{\text {pdm }}\right)$.

\subsection{Antigenic Analysis}

Hemagglutination inhibition (HI) assays were conducted as described [20]. Briefly, all sera were pre-treated with neuraminidase (Sigma, EC3.2.1.18 Type IV from Clostridium perfringens, 14-18 h at $\left.37^{\circ} \mathrm{C}\right)$; then sodium citrate $\left(1.5 \%\right.$ ) was added and inactivation was carried out for $30 \mathrm{~min}$ at $56{ }^{\circ} \mathrm{C}$, followed by adsorption to chicken erythrocytes for $1 \mathrm{~h}$ at $4-8{ }^{\circ} \mathrm{C}$. Antigens were adjusted to eight hemagglutinating units (HU). To carry out the test, the sera (already pre-diluted 1:10 through the pretreatment) were titrated in microtitre plates $(\log 2)$. The same volume of antigen suspension $(25 \mu \mathrm{L})$ adjusted to $8 \mathrm{HU}$ was pipetted into each of the wells of the microtitre plate and the mixture incubated for $30 \mathrm{~min}$ at room temperature. Then, $50 \mu \mathrm{L}$ of a $0.5 \%$ chicken erythrocyte suspension was added and the plates were allowed to stand for $30 \mathrm{~min}$ at room temperature.

Hyperimmune and immune sera in pigs were established and hemagglutination inhibition assays (HI) were performed as described previously $[13,15]$. In short, immune sera were generated by twofold immunization of 10 pigs with inactivated and Carbopol-adjuvanted virus of each

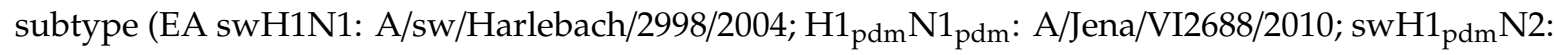
A/sw/Papenburg/12653/2010; swH1 pdm $_{\text {N1 }}$ pdm: A/sw/Wachtum/20657/2014) within 21 days; blood was taken 10 days after second immunization. The sera were pooled and tested against the virus isolates by HI. Hyperimmune sera were established by four-fold immunization $(0,14,28$ and 54 days after first shot) of one pig each (intramuscularly) with 64 hemagglutinating units of the corresponding inactivated virus adjuvanted with Freund's adjuvant (Sigma-Aldrich) or mineral oil (ISA25, Seppic). Blood samples were taken 70 days after first administration of the immunogen. Animal experiments were approved by the Landesverwaltungsamt Sachsen-Anhalt (Az 42502-3-401, 42502-3-642Ä, 42502-3-743, 45502-3-579).

\section{Results}

The German swine influenza surveillance, 2003-2015, was conducted by members of the FluResearchNet consortium and succeeded to collect 1310 S-IAV isolates. Our S-IAV archive was completed by 40 virus strains of other sources from 1979 to 2001 . These 40 virus strains plus 810 isolates of the surveillance have been sequenced by the Jena Swine Influenza Virus Sequencing Initiative, i.e., 348 swH1N1, 267 swH1N2, 229 swH3N2 and six swH3N1 isolates [8]. Genetic analysis of 348 swH1N1 isolates (328 of which were unpublished) revealed $304 \mathrm{EA}$ swH1N1 strains, $18 \mathrm{H} 1_{\text {pdm }} \mathrm{N} 1_{\text {pdm }}$ strains, 11 reassortants with gene segment(s) of $\mathrm{H} 1_{\mathrm{pdm}} \mathrm{N} 1_{\mathrm{pdm}}, 14$ isolates of the novel Schneiderkrug/2013-like swH1N1 reassortant and one classical swine (CS) H1N1 (Figure 1). 


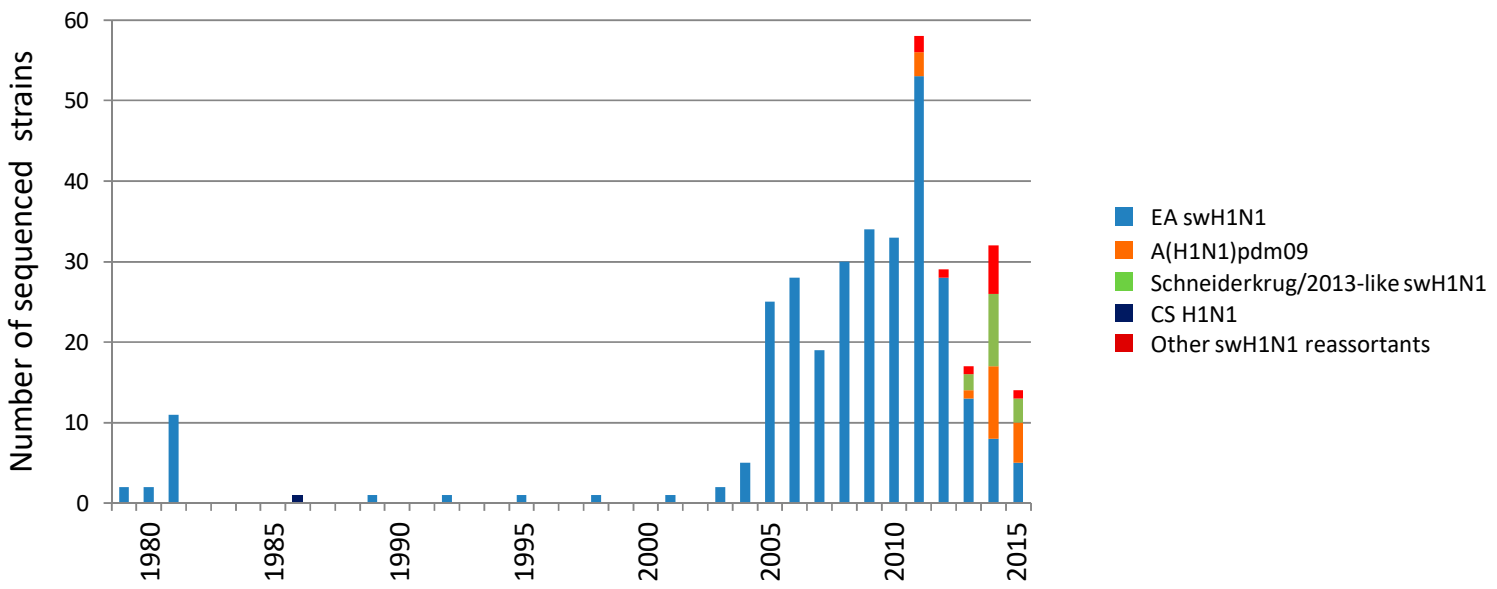

Year of isolation

Figure 1. Distribution of swH1N1 isolates. Year of isolation of H1N1 strains sequenced in this study. Different clades are indicated.

\subsection{Phylogenetic Analysis of German swH1N1 Isolates}

Phylogenetic analysis revealed that the HA gene of German swH1N1 isolates belongs to three co-circulating HAH1 lineages, named 1A, 1B and 1C according to the Anderson-2016 nomenclature (Figure 2a, Supplementary Figure S1). The HA gene of the prevalent EA swH1N1 strains belongs to lineage $1 \mathrm{C}$, whereas the $\mathrm{HA}$ of the $\mathrm{H} 1_{\mathrm{pdm}} \mathrm{N} 1_{\mathrm{pdm}}$ belongs to lineage $1 \mathrm{~A}$ and the Schneiderkrug/2013-like swH1N1 to lineage 1B. The latter viruses emerged in February 2013 in northwest Germany and have been detected in three German States (Figure 3). The viruses persisted until the end of the surveillance in December 2015. Five sublineages of 1C have been defined by Anderson et al. on basis of phylogenetic clustering: (i) Sublineage 1C.1 comprises the continental swH1N1 isolates of 1979-2000 and all English isolates, 1992-2011. (ii) Sublineage 1C.2 includes a few Danish and German swH1N1 strains, 1993-2013, plus the Emmelsbuell/2009-like swH1N2 and the Gladau/2012-like swH1N2. (iii) Sublineage 1C.2.1 contains swH1N1 strains of 2005-2015, (iv) sublineage 1C.2.2 strains of 2001-2014, and (v) sublineage 1C.2.3 five strains of 1999-2004. Beside phylogenetic clustering, no biological, geographical or other correlates could be identified that supported sub-classification of the S-IAV HA 1C lineage.

S-IAV strains with $\mathrm{HA}$ of lineage $1 \mathrm{~B}$ are derivatives of $\mathrm{H} 1_{\text {seas }} \mathrm{N} 1_{\text {seas, }}$, which disappeared after the 2009 pandemic. The swH1N2 strains of lineage 1B have been described recently [8]. Sublineage 1B.1.2.1 includes the continental swH1N2 (Gent/1999-like swH1N2) and two reassortants, the Diepholz/2008-like swH1N2 and the Schneiderkrug/2013-like swH1N1. Six segments of the latter reassortant (HA, PB1, PB2, PA, NP, M) are derived from the Diepholz/2008-like swH1N2, whereas the NA and NS segments originated from EA swH1N1.

Lineage $1 \mathrm{~A}$ includes $\mathrm{H} 1_{\mathrm{pdm}} \mathrm{N} 1_{\mathrm{pdm}}$ and derived $\mathrm{H} 1_{\mathrm{pdm}} \mathrm{N} 2$ strains (sublineage 1A.3.3.2). Whereas incursion of $\mathrm{H} 1_{\mathrm{pdm}} \mathrm{N} 1_{\mathrm{pdm}}$ into pigs has been observed several times since 2009 and persistent intra-herd transmission of S-IAV is a well-known phenomenon [29,30], farm-to-farm spread of $\mathrm{H} 1_{\mathrm{pdm}} \mathrm{N} 1_{\mathrm{pdm}}$ was not detected in the first years post-pandemic in Germany. In contrast, the Papenburg/2010-like swH1N2 soon became enzootic in Germany [13]. This situation changed in 2014, as the HA tree exhibits a cluster of 10 German sequences with greater 0.5 substitutions/site, which is exceptional (Figure 2a and Supplementary Figure S1). This result was also observed in the NA tree (Figure 2b and Supplementary Figure S2). This finding was confirmed in a refined phylogenetic analysis of HA which comprised 92 human $\mathrm{H} 1_{\mathrm{pdm}} \mathrm{N} 1_{\mathrm{pdm}}$ strains, $67 \mathrm{swH} 1_{\mathrm{pdm}} \mathrm{N} 1_{\mathrm{pdm}}, 86 \mathrm{swH} 1_{\mathrm{pdm}} \mathrm{N} 2$ and 13 reference sequences (CS H1N1 and North American triple reassortant H1N1) (Supplementary Figure S3). This group of German $\mathrm{swH} 1_{\mathrm{pdm}} \mathrm{N} 1_{\mathrm{pdm}}$ was named Wachtum/2014-like viruses after its first isolate and belonged to genogroup 6 according to WHO nomenclature (September 2018 interim report of the Worldwide Influenza Centre; https://www.crick.ac.uk/partnerships/worldwide-influenza-centre/annual-and-interim-reports, 
accessed 12 September 2019). These viruses exhibited up to 6\% diversity ( $p$-distance) to other human and porcine $\mathrm{H} 1_{\mathrm{pdm}} \mathrm{N} 1_{\mathrm{pdm}}$ strains (data not shown). The first isolate emerged in August 2014 in Lower Saxony in northwest Germany and spread to the neighboring States Northrhine Westphalia and Schleswig-Holstein (Figure 4) but also to Italy (Supplementary Figure S3). In addition, one H1N2 reassortant with a Wachtum/2014-like HA gene was also isolated.

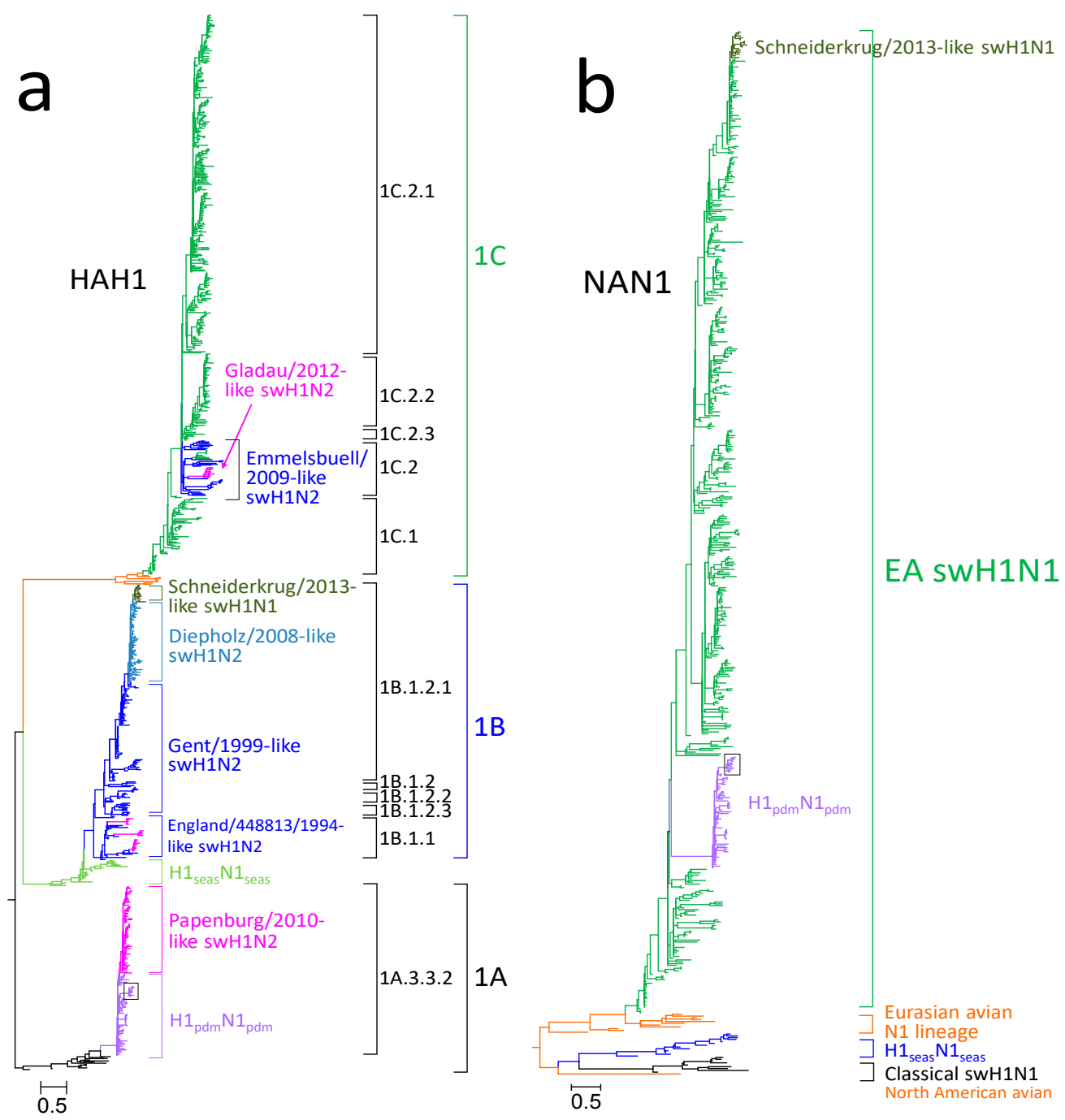

Figure 2. Phylogenetic analyses of hemagglutinin (HA) and neuraminidase (NA) genes using Bayesian tree inference. Relevant lineages and sublineages are indicated. The scale bars indicate substitutions per site. The Wachtum/2014-like $\mathrm{swH} 1_{\mathrm{pdm}} \mathrm{N1} 1_{\mathrm{pdm}}$ are boxed. (a) Analysis of $950 \mathrm{HAH} 1$ sequences of lineages 1A, 1B and 1C (Anderson-2016 nomenclature). Details are presented in Supplementary Figure S1. (b) Analysis of 577 NAN1 sequences. Details are presented in Supplementary Figure S2. Color code: green, EA swH1N1; light green, seasonal H1N1; olive, Schneiderkrug/2013-like swH1N1; purple, $\mathrm{H} 1_{\text {pdm }} \mathrm{N} 1_{\text {pdm }}$; magenta, $\mathrm{H}_{\text {pdm }} \mathrm{N} 2$ reassortants; blue, swH1N2; light blue, Diepholz/2008-like swH1N2; black, classical swine H1N1; ochre, avian H1. 


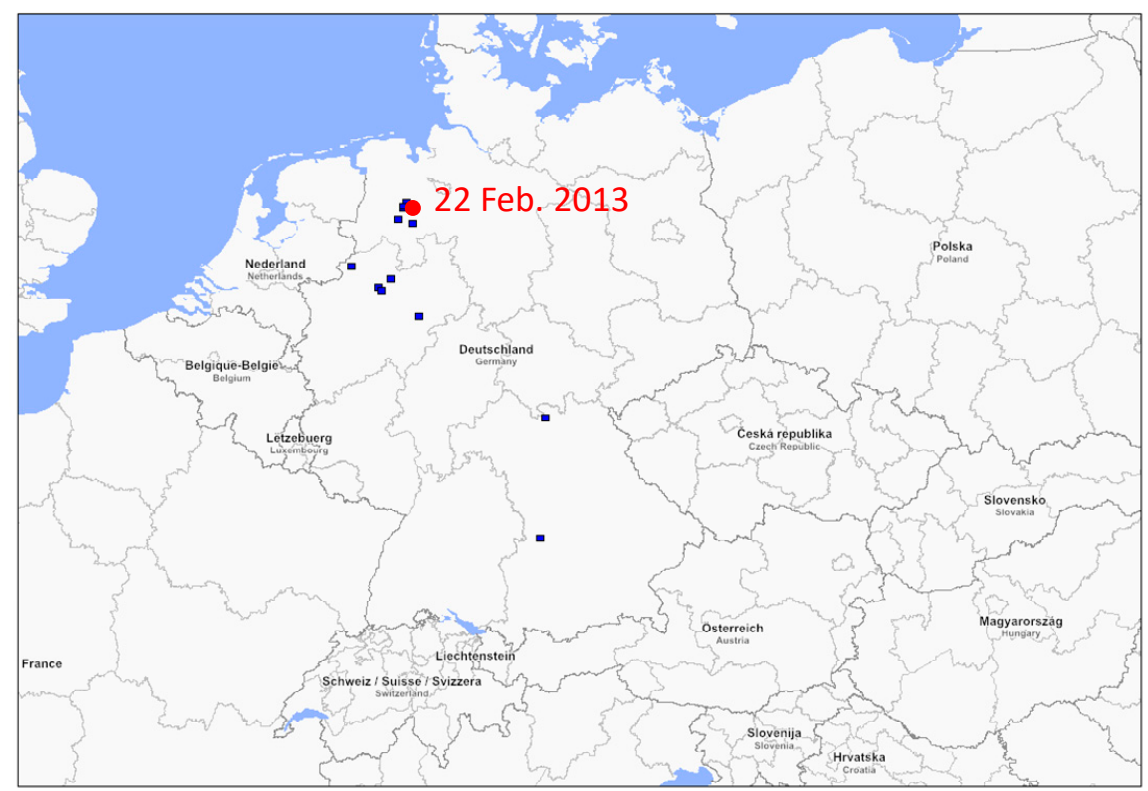

Figure 3. Distribution of Schneiderkrug/2013-like swH1N1 in Germany. Place and collection date of the first isolate is indicated.

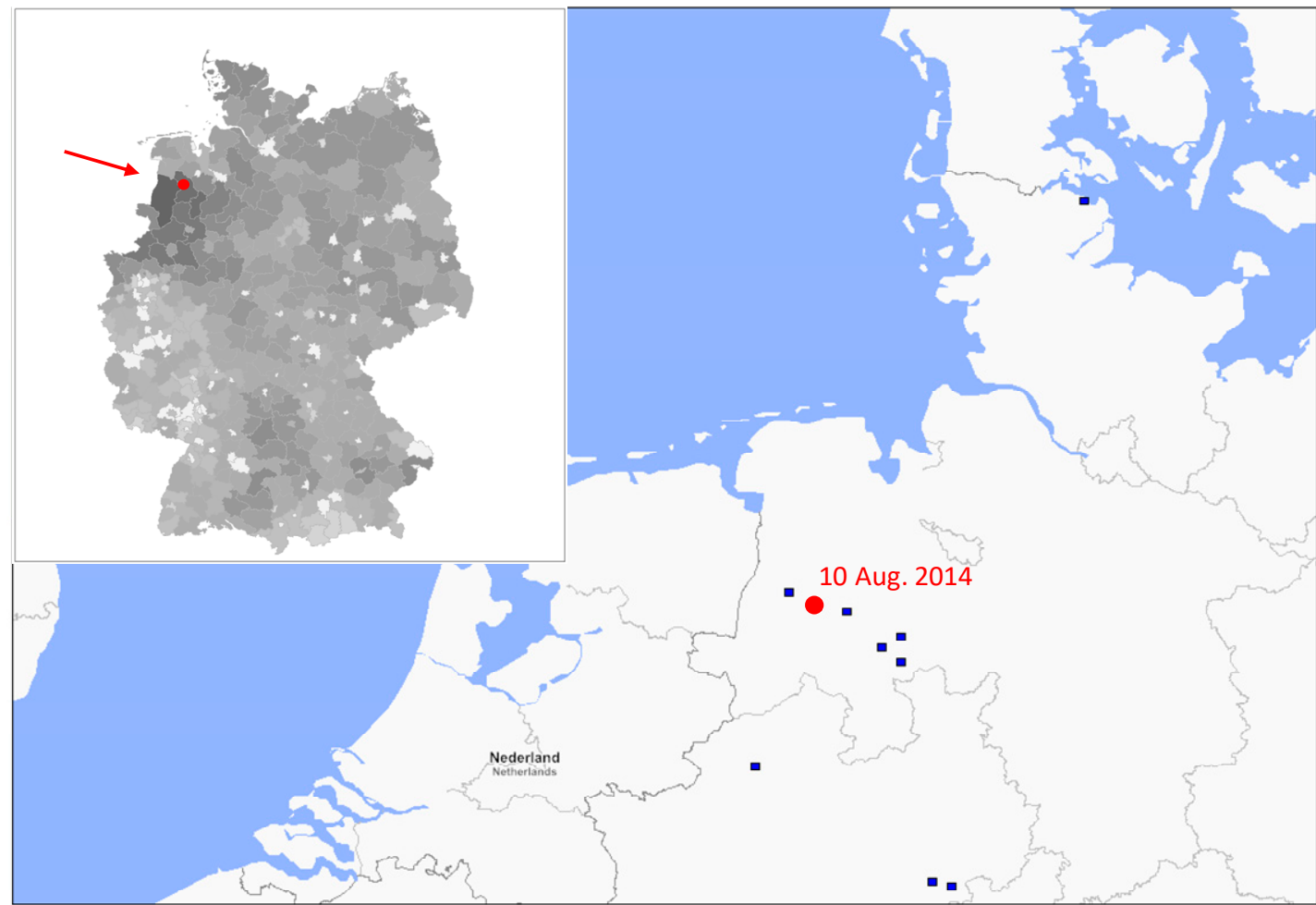

Figure 4. Incursion of Wachtum/2014-like $\mathrm{swH}_{\mathrm{pdm}} \mathrm{N} 1_{\mathrm{pdm}}$ into the German pig population. Dark squares indicate the places where isolates were collected; the place and sampling date of the first German isolate is printed in red. The inset presents the pig population density in Germany (gray shades correspond to the numbers of pigs per administrative district). The data were retrieved from the 2017-yearbooks of the 16 State Statistical Offices in Germany. 


\subsection{Analysis of Antigenic Sites of swH1N1}

For analysis of the antigenic sites as defined by Caton and Brownlee [31] and Brownlee and Fodor [32], we compared aa 183-187/220-222/252-254 (site Ca1), aa 154-159/238-239 (site Ca2), aa 87-92 (site Cb), aa 141-142/170-181 (site Sa) and aa 201-212 (site Sb) of lineages 1A, 1B and 1C (Anderson-2016 nomenclature; see Supplementary Figure S5A-C).

Phylogenetic analysis demonstrates that the NA gene of all EA swH1N1, $\mathrm{H} 1_{\mathrm{pdm}} \mathrm{N} 1_{\mathrm{pdm}}$ and Schneiderkrug/2013-like swH1N1 belong to the same clade of the Eurasian avian N1 lineage, which emerged in 1979 (compare Figure 2b and Supplementary Figure S2). Comparison of the HAH1 and NAN1 trees, however, revealed traces of previous intratypic reassortment events (see Supplementary Figure S4). We have identified 15 sequence clusters which persisted between six and 21 years. Several HA clusters (e.g., $\# 4, \# 8, \# 9, \# 10$ ) are scattered in the NA tree, which is compatible with the concept of intratypic reassortment. Intratypic and intertypic reassortment is also evident from the phylogenetic trees of the internal genes (data not shown).

The $\mathrm{H} 1_{\mathrm{pdm}} \mathrm{N} 1_{\mathrm{pdm}}$ strains have been assigned into genogroups by the Worldwide Influenza Centre-WHO Collaborating Centre for Reference and Research on Influenza-The Francis Crick Institute on basis of partial HA sequences and their antigenic properties (https://www.crick.ac.uk/ partnerships/worldwide-influenza-centre/annual-and-interim-reports, accessed 12 September 2019). These genogroups not necessarily correlate with phylogenetic clustering of full-length HA trees as shown in Supplementary Figure S3. This phylogenetic tree presents $245 \mathrm{H} 1_{\mathrm{pdm}} \mathrm{N} 1_{\text {pdm }}$ sequences plus 15 reference sequences of human and porcine strains. It demonstrates numerous independent incursions of $\mathrm{H} 1_{\mathrm{pdm}} \mathrm{N} 1_{\mathrm{pdm}}$ into the pig population in Europe. Noticeable, only two of the many anthroponotic events achieved to establish persistent infection chains in pigs: one led to the emergence of the Papenburg/2010-like swH1N2 lineage $[8,13,16]$ and the other to the Wachtum/2014-like swH1 ${ }_{\mathrm{pdm}} \mathrm{N} 1_{\mathrm{pdm}}$. Whereas virus isolates of sporadic, non-persistent spill-over infections show only single amino acid substitutions at the antigenic sites, the Papenburg/2010-like and the Wachtum/2014-like strains exhibit numerous amino acid exchanges (Supplementary Figure S5C). Amino acid substitutions of both virus groups correlate with altered antigenic properties. For the Papenburg/2010-like swH1N2, the lack of cross-reactivity with EA swH1N1 has been shown to be due to five substitutions, K159R, G172E, I183V, S202N and D204S [13,16], but on the basis of 79 strains of this cluster, additional substitutions have been identified, e.g., A90S, N142D/K/S, P154S, A158T/E, L178I, K180I, D185G, G187R, A203D, S207T, Q210K, A212T and R222K. All Papenburg/2010-like strains exhibit 5-10 substitutions in antigenic sites. The Wachtum/2014-like viruses show 11 amino acid substitutions in antigenic sites: P141T, N142D, K159S, G172T, N173D, S179N, K180I, D185N, S202A, S207R and D239N. In addition, both the Papenburg/2010-like and the Wachtum/2014-like viruses exhibit an additional potential glycosylation site at N179 and N202, respectively, which are located in antigenic sites Ca1 and Sb (Supplementary Figure S5C).

In order to investigate cross-reactivity of sporadic, anthroponotic $\mathrm{H} 1_{\mathrm{pdm}} \mathrm{N} 1_{\mathrm{pdm}}$ and Wachtum/2014-like swH1 $1_{\mathrm{pdm}} \mathrm{N} 1_{\mathrm{pdm}}$ viruses as well as EA swH1N1 and $\mathrm{swH} 1_{\mathrm{pdm}} \mathrm{N} 2$ viruses, immune sera and hyperimmune sera were established and tested in the HI assay. The data show different degrees in cross-reactivity but clearly indicate that the $\mathrm{swH}_{\mathrm{pdm}} \mathrm{N} 2$ as well as the Wachtum-2014-like $\mathrm{swH} 1_{\mathrm{pdm}} \mathrm{N} 1_{\mathrm{pdm}}$ viruses still exhibit only a very low cross-reactivity in hyperimmune sera to EA swH1N1 and human $\mathrm{H} 1_{\mathrm{pdm}} \mathrm{N} 1_{\mathrm{pdm}}$, whereas immune sera of Wachtum-2014-like $\mathrm{swH} 1_{\mathrm{pdm}} \mathrm{N} 1_{\mathrm{pdm}}$ viruses lack cross-reactivity to the other viruses (Table 1 ). The cross-reactive pattern between $\mathrm{swH} 11_{\mathrm{pdm}} \mathrm{N} 2$ and Wachtum-2014-like $\mathrm{H} 1_{\mathrm{pdm}} \mathrm{N} 1_{\mathrm{pdm}}$ is very low. Thus, two antigenic variant $\mathrm{H} 1_{\mathrm{pdm}} \mathrm{Nx}$ viruses circulate in German pigs. 
Table 1. Immunogenic pattern of Wachtum/2014-like $\mathrm{swH} 1_{\mathrm{pdm}} \mathrm{N} 1_{\mathrm{pdm}}$ in comparison to other H1N1 and $\mathrm{H} 1 \mathrm{~N} 2$ viruses detected in pigs in Germany (HI titer reciprocal).

\begin{tabular}{|c|c|c|c|c|}
\hline & EA swH1N1 & $\begin{array}{c}\text { Sporadic, Anthropogenic } \\
\qquad 1_{\mathrm{pdm}} \mathrm{N} 1_{\mathrm{pdm}}\end{array}$ & $\begin{array}{c}\text { Papenburg/2010-Like } \\
\text { H1 } 1_{\text {pdm }} \text { N2 }\end{array}$ & $\begin{array}{l}\text { Wachtum/2014-Like } \\
\text { H1 } 1_{\text {pdm }} N 1_{\text {pdm }}\end{array}$ \\
\hline \multicolumn{5}{|c|}{ Immune Sera } \\
\hline EA swH1N1 & 197 & $<20$ & $<20$ & $<20$ \\
\hline anthropogenic $\mathrm{H} 1_{\mathrm{pdm}} \mathrm{N} 1_{\mathrm{pdm}}$ & 21 & 106 & 34 & $<20$ \\
\hline Papenburg/2010-like $\mathrm{H} 1_{\mathrm{pdm}} \mathrm{N} 2$ & $<20$ & 49 & 485 & $<20$ \\
\hline Wachtum/2014-like $\mathrm{H} 1_{\mathrm{pdm}} \mathrm{N} 1_{\mathrm{pdm}}$ & $<20$ & $<20$ & $<20$ & 160 \\
\hline \multicolumn{5}{|c|}{ Hyper Immune Sera } \\
\hline EA swH1N1 & 2560 & 80 & 160 & 80 \\
\hline anthropogenic $\mathrm{H}_{\mathrm{pdm}} \mathrm{N} 1_{\mathrm{pdm}}$ & 640 & 2560 & 640 & 320 \\
\hline Papenburg/2010-like $\mathrm{H}_{\mathrm{pdm}} \mathrm{N} 2$ & 80 & 320 & 2560 & 20 \\
\hline Wachtum/2014-like $\mathrm{H} 1_{\mathrm{pdm}} \mathrm{N} 1_{\mathrm{pdm}}$ & 160 & 40 & 20 & 2560 \\
\hline
\end{tabular}

The Schneiderkrug/2013-like swH1N1 are antigenically very similar to their parental Diepholz/2008-like swH1N2, i.e., they are characterized by P141S/K142N/T174I of antigenic site Sa (Supplementary Figure S5B).

Analysis of N-glycosylation patterns of HA proteins revealed three groups in the 1A lineage, which correspond to the sporadic anthroponotic $\mathrm{H}_{\mathrm{pdm}} \mathrm{N1}_{\mathrm{pdm}}$, the Wachtum/2014-like viruses and the Papenburg/2010-like swH1N2 (Table 2). Furthermore, three patterns of 1B viruses are distinguishable: English swH1N2, Gent/1999-like swH1N2 and the Diepholz/2008-like swH1N2 together with the Schneiderkrug/2017-like swH1N1. Less conclusive is the distinction of the strains of lineage 1C. The viruses of sublineage $1 \mathrm{C} .2$ and its sub-sublineages (1C.2.1, 1C.2.2, 1C.2.3) comprise one group but some variation is seen in up to four $\mathrm{N}$-glycosylation sequons. Viruses of sublineage 1C.1 fall into two groups, the early isolates of 1979-1989, which are like avian H1N1, and the late strains isolated from 1991 onwards, which include virus isolates from the continent and the British Isles. 
Table 2. N-Glycosylation pattern of $\mathrm{H} 1$ of European S-IAV.

\begin{tabular}{|c|c|c|c|c|c|c|c|c|c|c|c|c|c|c|c|c|c|c|}
\hline & \multicolumn{18}{|c|}{ Amino Acid Position of Glycosylation Sequons (N-X-S/T) } \\
\hline & $27-29$ & $28-30$ & $40-42$ & $101-103$ & 104-106 & $136-138$ & $142-144^{1}$ & $172-174^{1}$ & $177-179^{1}$ & $179-181^{2}$ & $202-204^{3}$ & $212-214$ & $286-288$ & $291-293$ & 293-295 & $304-306$ & $498-500$ & $557-559$ \\
\hline \multicolumn{19}{|c|}{ Lineage 1A } \\
\hline anthroponotic $\mathrm{H}_{\mathrm{pdm}} \mathrm{N} 1_{\mathrm{pdm}}$ & + & + & + & - & + & - & - & - & - & - & - & - & - & - & + & + & + & + \\
\hline Wachtum/2014-like $s w H 1_{\text {pdm }} \mathrm{N1}_{\mathrm{pdm}}$ & + & + & + & - & + & - & - & - & - & + & - & - & - & - & + & + & + & + \\
\hline Papenburg/2010-like swH1N2 & + & + & + & - & + & - & - & - & - & - & + & - & - & - & + & + & + & + \\
\hline \multicolumn{19}{|c|}{ Lineage 1B } \\
\hline Schneiderkrug/2014-like swH1N1 & + & + & + & - & - & - & + & - & + & - & - & - & + & - & - & + & + & + \\
\hline Diepholz/2008-like swH1N2 & + & + & + & - & - & - & + & - & + & - & - & - & + & - & - & + & + & + \\
\hline Gent/1999-like swH1N2 & + & + & + & - & - & - & - & + & + & - & - & - & + & - & - & + & + & + \\
\hline England/448813/1994-like swH1N2 & + & + & + & - & - & - & - & - & + & - & - & - & + & - & - & + & + & + \\
\hline \multicolumn{19}{|c|}{ Lineage 1C } \\
\hline $\begin{array}{l}\text { sublineage 1C.2 (EA swH1N1, } \\
\text { swH1N2) }\end{array}$ & + & + & + & B & B & B & - & - & - & B & - & + & - & + & - & - & + & + \\
\hline sublineage 1C.1, 1991-1998 & + & + & + & - & + & - & - & - & - & B & - & - & - & + & - & - & + & + \\
\hline sublineage 1C.1, 1979-1989 & + & + & + & - & + & - & - & - & - & - & - & - & - & - & - & + & + & + \\
\hline avian H1N1 & + & + & + & - & + & - & - & - & - & - & & - & - & - & - & + & + & + \\
\hline
\end{tabular}

${ }^{1}$ part of antigenic site $\mathrm{Sa},{ }^{2}$ part of antigenic site $\mathrm{Ca1},{ }^{3}$ part of antigenic site $\mathrm{Sb}, \mathrm{B}$, both variants (with and without glycosylation sequons) are observed. 


\section{Discussion}

Swine influenza is enzootic in Germany and many other European countries. A recent German 13-year long-term swine influenza surveillance conducted by the German FluResearchNet consortium revealed significant changes in the prevalence of circulating swH1N2 strains [8]. From 2008 onwards, several novel reassortants emerged in Germany, established stable infection chains and now co-circulate with pre-existing S-IAVs (Figure 5). In the present study, we focused on swH1N1 and made two remarkable observations: (1) a sustained circulation of swine-adapted $\mathrm{H} 1_{\mathrm{pdm}} \mathrm{N} 1_{\text {pdm }}$, named Wachtum/2014-like swH1 $1_{\mathrm{pdm}} \mathrm{N} 1_{\mathrm{pdm}}$, which is a drift variant, and (2) emergence of a novel swH1N1 $6+2$ reassortant. This reassortant, designated Schneiderkrug/2013-like virus, is a derivative of the Diepholz/2008-like swH1N2 [8] and of an EA swH1N1 (donor of NA and NS). It is serologically inconspicuous, spread to three German States and co-circulates with the EA swH1N1 and the $\mathrm{H} 1_{\text {pdm }} \mathrm{N} 1_{\text {pdm }}$. This is interesting as the HA of these three viruses belong to three different genetic lineages, named 1A, 1B and 1C (compare Figure 2A), and hence, are antigenically heterologous. In addition, each of the three swH1N1 viruses co-circulates with swH1N2 viruses of the same HA sublineage. The respective co-circulating viruses are: (i) lineage 1 A: Wachtum/2014-like swH1 $1_{\text {pdm }} 1_{\text {pdm }}$ and Papenburg/2010-like swH1N2, (ii) lineage 1B: Schneiderkrug/2013-like swH1N1 and Diepholz/2008-like swH1N2 and (iii) lineage 1C: Emmelsbuell/2009-like plus Gladau/2012-like swH1N2 and the EA swH1N1. Different antigenic properties of the Wachtum/2014-like swH1 $1_{\text {pdm }} \mathrm{N} 1_{\text {pdm }}$ have been demonstrated in HI assays and correlate with specific substitutions of antigenic sites (Supplementary Figure S5C). Moreover, we observed clade-specific glycosylation patterns of the HA (Table 2) as evident from conserved/non-conserved NxS/T glycosylation sequons. Different $\mathrm{N}$-glycosylation patterns may influence antigenic properties, binding to receptors, the HA/NA balance and transmissibility of the respective virus [33-35].

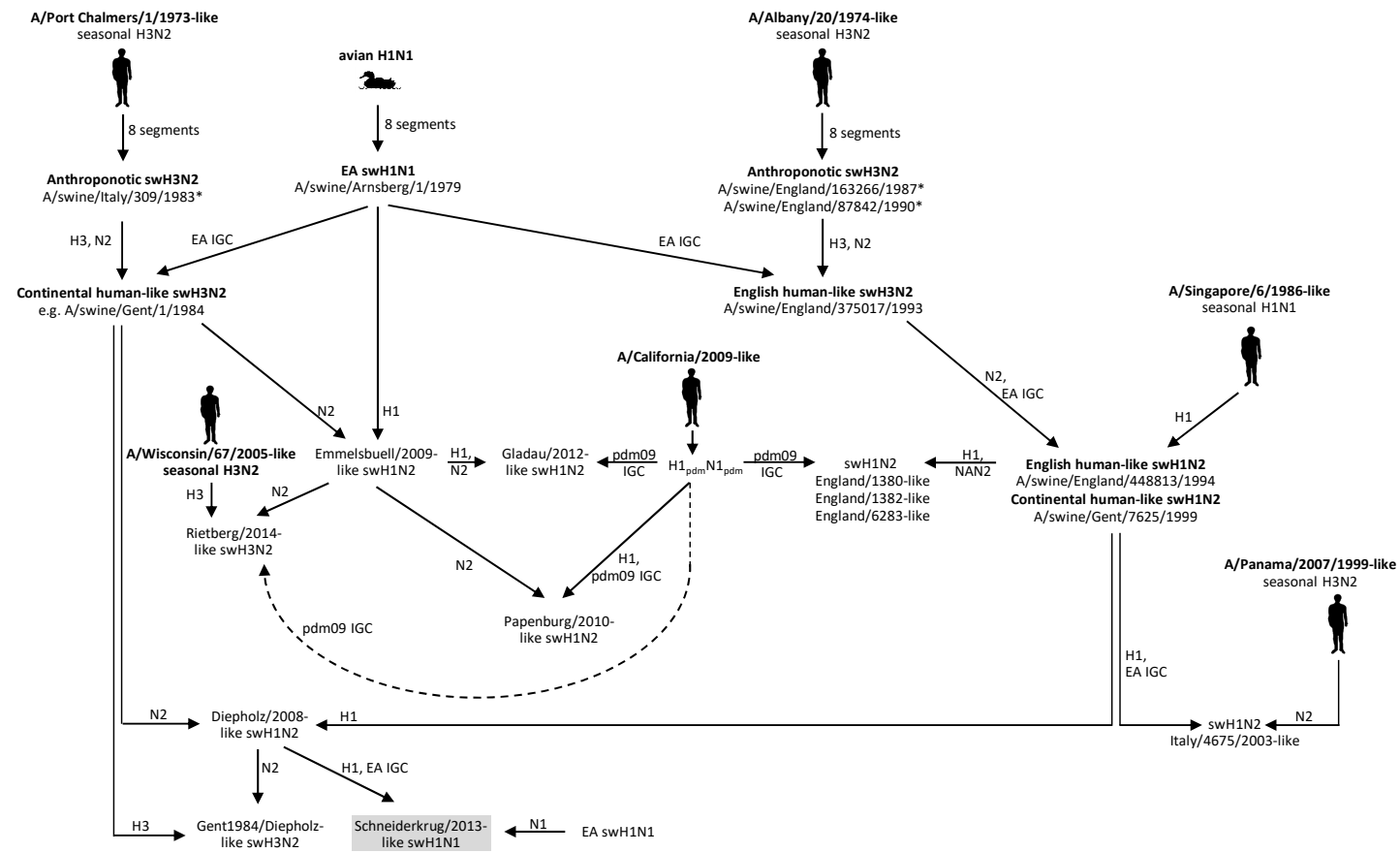

Figure 5. Evolution of German S-IAVs. Reassortment events leading to the main S-IAV lineages and the novel swH1N1 virus (presented in gray box) are shown. The Wachtum/2014-like swH1N1 are not shown here. Pictograms denote anthroponotic and spill-over infections. Asteriscs $\left(^{*}\right)$ indicate isolates with partial sequences.

EA swH1N1 is prevalent in Germany and is known to cross-react at a certain level with $\mathrm{H} 1_{\mathrm{pdm}} \mathrm{N} 1_{\mathrm{pdm}}$. This cross-reactivity may have prevented a sustained circulation of $\mathrm{H} 1_{\mathrm{pdm}} \mathrm{N} 1_{\mathrm{pdm}}$ in pigs 
for several years, especially in pig herds that had contact with several EA swH1N1 viruses and therefore exhibited a broader cross-reactivity [15]. Sporadic anthropogenic infections of pigs with $\mathrm{H}_{\mathrm{pdm}} \mathrm{N} 1_{\mathrm{pdm}}$ occurred in Germany, mostly in areas with low-dense pig population, but no sustained circulation was observed. This changed with the upsurge of the Papenburg/2010-like swH1N2. Whereas the previous isolates of this reassortant exhibited five amino acid exchanges in their antigenic sites [13,16], the more recent strains have accumulated up to 10 amino acid substitutions (Supplementary Figure S5C). In 2014, the Wachtum/2014-like swH1 ${ }_{\mathrm{pdm}} \mathrm{N} 1_{\mathrm{pdm}}$ emerged with 11 amino acid substitutions in the antigenic sites plus an additional $\mathrm{N}$-glycosylation site (Table 1 ). Whereas the Papenburg/2010-like viruses still display a very low degree of cross-reactivity to the human $\mathrm{H} 1_{\mathrm{pdm}} \mathrm{N} 1_{\mathrm{pdm}}$, the Wachtum/2014-like $\mathrm{swH} 1_{\mathrm{pdm}} \mathrm{N} 1_{\mathrm{pdm}}$ viruses neither cross-react with the EA swH1N1 nor with other $\mathrm{H} 1_{\mathrm{pdm}} \mathrm{N} 1_{\mathrm{pdm}}$ in immune sera. Hence, these viruses were the first $\mathrm{H} 1_{\mathrm{pdm}} \mathrm{N} 1_{\mathrm{pdm}}$ that accomplished a persistent infection chain in pigs. This feature may bear future problems in the pig industry, as no commercial vaccine is available to protect pigs sufficiently from this virus.

Notably, three mechanisms of S-IAV immune escape are observed: (i) The emergence of drift variants, which are not adequately matched by the available vaccines (e.g., the Wachtum/2014-like $\mathrm{swH} 1_{\mathrm{pdm}} \mathrm{N} 1_{\mathrm{pdm}}$ ), (ii) the emergence of reassortants and (iii) a combination of reassortment and antigenic drift (e.g., the Papenburg/2010-like swH1N2 [13]). The appearance of new S-IAV variants in quick succession make it increasingly difficult to ensure an all-encompassing vaccination of pigs. Beside novel swH1N1 variants, the diversity of the swH1N2 viruses has also increased in the last decade in Germany [8]. It is likely that changed swine farming practices and increased herd size as well as the invention of a trivalent swine influenza vaccine in Germany contributed to this diversity. Rose et al. and Ryt-Hansen et al. described increasing problems in herd management due to persistent S-IAV infection in both farrow-to-finish and farrow-to-nursery farms [29,30]. Only a few modeling studies addressed S-IAV dynamics in pig herds. Reynolds et al. investigated different vaccination strategies and indicated the failure to eliminate swine influenza if only partial protection is achieved by the vaccine due to circulating heterologous S-IAV strains [36]. Cador et al. demonstrated that the presence of maternally derived antibodies may lead to an extended duration of epidemics and batch-to-batch infection [37]. The problem is further aggravated by epidemiological models that had been calibrated against seroprevalence data from Dutch finishing pigs and pointed out that S-IAV may persist even in small pig herds [38]. The co-circulation of two distinct S-IAV strains was investigated in another study of Cador et al. [39]. Modeling different vaccination schemes and herd management strategies, these authors evaluated the probability of a co-infection event in France to circa 17\%, and no vaccination strategy achieved S-IAV elimination within the farrow-to-finish pig herd.

These studies are compatible with our observations: repeated virus isolation within the same farm, isolation of different types and the emergence of reassortants are indicative of persistent infection of a herd despite vaccination. The unexpected occurrence of the Wachtum/2014-like drift variant with 11 substitutions in the antigenic sites of HA is concerning, as this indicates long-lasting inconspicuous circulation afore. The observed spread of this variant to neighboring German states suggests increasing enzootic circulation. In addition, such a drift variant bears a certain zoonotic risk, as the human IAV vaccine probably does not cover its antigenic properties. Our data once more indicate the necessity to intensify broader swine influenza surveillance.

Supplementary Materials: The following are available online at http://www.mdpi.com/1999-4915/12/7/762/s1, Supplementary Information S1: Legends to supplementary figures, Figure S1: Phylogenetic analysis and genotypes of 950 HAH1 sequences, Figure S2: Phylogenetic analysis of 577 NAN1 sequences, Figure S3: Phylogenetic analysis of 258 HAH1 sequences of lineage 1A, Figure S4: Detection of intratypic reassortment, Figure S5: Analysis of antigenic sites, Table S1: List of investigated strains.

Author Contributions: Conceptualization, R.D. and R.Z.; investigation, R.D., M.G., A.K., J.L., A.P. and R.Z.; writing — original draft preparation, R.Z.; writing — review and editing, R.Z., A.K. and R.D.; funding acquisition, R.D. and R.Z. All authors have read and agreed to the published version of the manuscript. 
Funding: This research was funded by the Federal Ministry of Education and Research within the frame work of the FluResearchNet (grant nos. 01KI7142, 01KI07143 awarded to R.Z. and 01KI1006J awarded to R.D.) and by the European Union FP7-INFLUENZA-2010 ESNIP3 program (grant \#259949 awarded to R.D.).

Acknowledgments: The authors thank Martina Müller, Ivonne Heinze and Ivonne Görlich for technical assistance. Furthermore, we are indebted to Brunhilde Schweiger, Robert Koch-Institut, Berlin, for providing seasonal influenza viruses, Kristien van Reeth, Ghent University, for providing swine influenza viruses from 1992, members of FluResearchNet for providing influenza virus isolates and all veterinarians and swine holders who submitted samples to the partner laboratories and Franz Lappe, Geseke, for frequent submission of samples and continued discussion on swine influenza in the field.

Conflicts of Interest: The authors declare no conflict of interest. The funders had no role in the design of the study; in the collection, analyses or interpretation of data; in the writing of the manuscript, or in the decision to publish the results.

\section{References}

1. Mena, I.; Nelson, M.I.; Quezada-Monroy, F.; Dutta, J.; Cortes-Fernandez, R.; Lara-Puente, J.H.; Castro-Peralta, F.; Cunha, L.F.; Trovao, N.S.; Lozano-Dubernard, B.; et al. Origins of the 2009 H1N1 influenza pandemic in swine in Mexico. eLife 2016, 5, e16777. [CrossRef]

2. Pensaert, M.; Ottis, K.; Vandeputte, J.; Kaplan, M.M.; Bachmann, P.A. Evidence for the natural transmission of influenza A virus from wild ducks to swine and its potential importance for man. Bull. WHO 1981, 59, 75-78.

3. Witte, K.K.; Nienhoff, H.; Ernst, H.; Schmidt, U.; Prager, D. Erstmaliges Auftreten einer durch das Schweineinfluenzavirus verursachten Epizootie in Schweinebeständen der Bundesrepublik Deutschland. Tierärztliche Umsch. 1981, 36, 591-606, [In German].

4. Zell, R.; Scholtissek, C.; Ludwig, S. Genetics, evolution, and the zoonotic capacity of European swine influenza viruses. Curr. Top. Microbiol. Immunol. 2013, 370, 29-55. [PubMed]

5. Choi, Y.K.; Pascua, P.N.Q.; Song, M.S. Swine influenza viruses: An Asian perspective. Curr. Top. Microbiol. Immunol. 2013, 370, 147-172. [PubMed]

6. Krumbholz, A.; Lange, J.; Sauerbrei, A.; Groth, M.; Platzer, M.; Kanrai, P.; Pleschka, S.; Scholtissek, C.; Büttner, M.; Dürrwald, R.; et al. The origin of the European avian-like swine influenza viruses. J. Gen. Virol. 2014, 95, 2372-2376. [CrossRef] [PubMed]

7. Watson, S.; Langat, P.; Reid, S.M.; Lam, T.T.Y.; Cotton, M.; Kelly, M.; Van Reeth, K.; Qiu, Y.; Simon, G.; Bonin, E.; et al. Molecular epidemiology and evolution of influenza viruses circulating within European swine between 2009 and 2013. J. Virol. 2015, 89, 9920-9931. [CrossRef]

8. Zell, R.; Groth, M.; Krumbholz, A.; Lange, J.; Philipps, A.; Dürrwald, R. Displacement of the Gent/1999 human-like swine H1N2 influenza A virus lineage by novel reassortant H1N2 clades in Gemany. Arch. Virol. 2020, 165, 55-67. [CrossRef] [PubMed]

9. Moreno, A.; Chiapponi, C.; Boniotti, M.B.; Sozzi, E.; Foni, E.; Barbieri, I.; Zanoni, M.G.; Faccini, S.; Lelli, D.; Cordioli, P. Genomic characterization of H1N2 swine influenza viruses in Italy. Vet. Microbiol. 2012, 156, 265-276. [CrossRef]

10. Hofshagen, M.; Gjerset, B.; Er, C.; Tarpai, A.; Brun, E.; Dannevig, B.; Bruheim, T.; Fostad, I.G.; Iversen, B.; Hungnes, O.; et al. Pandemic influenza A(H1N1)v: Human to pig transmission in Norway? Eurosurveillance 2009, 14, 19406. [CrossRef]

11. Welsh, M.D.; Baird, P.M.; Guelbenzu-Gonzalo, M.P.; Hanna, A.; Reid, S.M.; Essen, S.; Russell, C.; Thomas, S.; Barrass, L.; McNeilly, F.; et al. Initial incursion of pandemic (H1N1) 2009 influenza A virus into European pigs. Vet. Rec. 2010, 166, 642-645. [CrossRef]

12. Moreno, A.; Di Trani, L.; Alborali, L.; Vaccari, G.; Barbieri, I.; Falcone, E.; Sozzi, E.; Puzelli, S.; Ferri, G.; Cordioli, P. First pandemic H1N1 outbreak from a pig farm in Italy. Open Virol. J. 2010, 4, 52-56. [CrossRef]

13. Lange, J.; Groth, M.; Schlegel, M.; Krumbholz, A.; Wieczorek, K.; Ulrich, R.; Köppen, S.; Schulz, K.; Appl, D.; Selbitz, H.J.; et al. Reassortment of the pandemic (H1N1) 2009 virus and establishment of a novel porcine H1N2 influenza virus lineage in Germany. Vet. Microbiol. 2013, 167, 345-356. [CrossRef] [PubMed]

14. Sokolov, I.; Kurskaya, O.; Leonov, S.; Kabilov, M.; Alikina, T.; Alekseev, A.; Yushkov, Y.; Saito, T.; Uchida, Y.; Mine, J.; et al. Novel reassortant H1N1 swine influenza virus detected in pig population in Russia. Emerg. Microbes Inf. 2019, 8, 1. 
15. Dürrwald, R.; Krumbholz, A.; Baumgarte, S.; Schlegel, M.; Vahlenkamp, T.W.; Selbitz, H.J.; Wutzler, P.; Zell, R. Swine influenza A vaccines, pandemic (H1N1) 2009 virus, and cross-reactivity. Emerg. Infect. Dis. 2010, 16, 1029-1030. [CrossRef] [PubMed]

16. Harder, T.C.; Grosse Beilage, E.; Lange, E.; Meiners, C.; Döhring, S.; Pesch, S.; Noe, T.; Grund, C.; Beer, M.; Starick, E. Expanded cocirculation of sTable Subtypes, emerging lineages, and new sporadic reassortants of porcine influenza viruses in swine populations in Northwest Germany. J. Virol. 2013, 87, 10460-10476. [CrossRef] [PubMed]

17. Simon, G.; Larsen, L.E.; Dürrwald, R.; Foni, E.; Harder, T.; Van Reeth, K.; Markowska-Daniel, I.; Reid, S.M.; Dan, A.; Maldonado, J.; et al. European suveillance network for influenza in pigs: Surveillance programs, diagnostic tools and swine influenza virus subtypes identified in 14 European countries from 2010 to 2013. PLoS ONE 2014, 9, e115815. [CrossRef]

18. Lange, J.; Groth, M.; Kanrai, P.; Pleschka, S.; Scholtissek, C.; Dürrwald, R.; Platze, M.; Sauerbrei, A.; Zell, R. Circulation of classical swine influenza virus in Europe between the wars? Arch. Virol. 2014, 159, 1467-1473. [CrossRef]

19. Fouchier, R.A.; Bestebroer, T.M.; Herfst, S.; Van Der Kemp, L.; Rimmelzwaan, G.F.; Osterhaus, A.D. Detection of influenza A viruses from different species by PCR amplification of conserved sequences in the matrix gene. J. Clin. Microbiol. 2000, 38, 4096-4101. [CrossRef]

20. Zell, R.; Bergmann, S.; Krumbholz, A.; Wutzler, P.; Dürrwald, R. Ongoing evolution of swine influenza viruses: A novel reassortant. Arch. Virol. 2008, 153, 2085-2092. [CrossRef]

21. Hoffmann, E.; Stech, J.; Guan, Y.; Webster, R.G.; Perez, D.R. Universal primer set for the full-length amplification of all influenza A viruses. Arch. Virol. 2001, 146, 2275-2289. [CrossRef] [PubMed]

22. Stech, J.; Stech, O.; Herwig, A.; Altmeppen, H.; Hundt, J.; Gohrbandt, S.; Kreibich, A.; Weber, S.; Klenk, H.D.; Mettenleiter, T.C. Rapid and reliable universal cloning of influenza A virus genes by target-primed plasmid amplification. Nucl. Acids Res. 2008, 36, e139. [CrossRef]

23. Groth, M.; Lange, J.; Kanrai, P.; Pleschka, S.; Scholtissek, C.; Krumbholz, A.; Platzer, M.; Sauerbrei, A.; Zell, R. The genome of an influenza virus from a pilot whale: Relation to influenza viruses of gulls and marine mammals. Inf. Genet. Evol. 2014, 24, 183-186. [CrossRef] [PubMed]

24. Tamura, K.; Peterson, D.; Peterson, N. MEGA5: Molecular evolutionary genetics analysis using maximum likelihood, evolutionary distance, and maximum parsimony methods. Mol. Biol. Evol. 2011, 28, 2731-2739. [CrossRef] [PubMed]

25. Ronquist, F.; Huelsenbeck, J.P. MrBayes 3: Bayesian phylogenetic inference under mixed models. Bioinformatics 2003, 19, 1572-1574. [CrossRef] [PubMed]

26. Drummond, A.J.; Suchard, M.A.; Xie, D.; Rambaut, A. Bayesian phylogenetics with BEAUTi and the BEAST 1.7. Mol. Biol. Evol. 2012, 29, 1969-1973. [CrossRef] [PubMed]

27. Kumar, S.; Stecher, G.; Li, M.; Knyaz, C.; Tamura, K. MEGA X: Molecular Evolutionary Genetics Analysis across computing platforms. Mol. Biol. Evol. 2018, 35, 1547-1549. [CrossRef]

28. Anderson, T.K.; Macken, C.A.; Lewis, N.S.; Scheuermann, R.H.; Van Reeth, K.; Brown, I.H.; Swenson, S.L.; Simon, G.; Saito, T.; Berhane, Y.; et al. A phylogeny-based global nomenclature system and automated annotation tool for $\mathrm{H} 1$ hemagglutinin genes from swine influenza A viruses. mSphere 2016, 1, e00275-16. [CrossRef]

29. Rose, N.; Herve, S.; Eveno, E.; Barbier, N.; Eono, F.; Dorenlor, V.; Andraud, M.; Camsusou, C.; Madec, F.; Simon, G. Dynamics of influenza A virus infections in permanently infected pig farms: Evidence of recurrent infections, circulation of several swine influenza viruses and reassortment events. Vet. Res. 2013, 44, 72. [CrossRef]

30. Ryt-Hansen, P.; Larsen, I.; Kristensen, C.S.; Krog, J.S.; Wachek, S.; Larsen, L.E. Longitudinal field studies reveal early infection and persistence of influenza A virus in piglets despite the presence of maternally derived antibodies. Vet. Res. 2019, 50,36. [CrossRef]

31. Caton, A.J.; Brownlee, G.G. The antigenic structure of the influenza virus A/PR/8/34 hemagglutinin (H1 subtype). Cell 1982, 31, 417-427. [CrossRef]

32. Brownlee, G.G.; Fodor, E. The predicted antigenicity of the haemagglutinin of the 1918 Spanish influenza pandemic suggests an avian origin. Philos. Trans. R. Soc. Lond. B Biol. Sci. 2001, 356, 1871-1876. [CrossRef] [PubMed] 
33. Huang, Y.; Owino, S.O.; Crevar, C.J.; Carter, D.M.; Ross, T.M. N-linked glycans and K147 residue on hemagglutinin synergize to elicit broadly reactive H1N1 influenza virus antibodies. J. Virol. 2020, 94, e01432-19. [CrossRef]

34. Linster, M.; van Boheemen, S.; de Graaf, M.; Schrauwen, E.J.A.; Lexmond, P.; Mänz, B.; Bestebroer, T.M.; Baumann, J.; van Riel, D.; Rimmelzwaan, G.F.; et al. Identification, characterization, and natural selection of mutations driving airborne transmission of A/H5N1 virus. Cell 2014, 157, 329-339. [CrossRef] [PubMed]

35. Park, S.; Lee, I.; Kim, J.I.; Bae, J.Y.; Yoo, K.; Kim, J.; Nam, M.; Park, M.; Yun, S.H.; Cho, Y.S.; et al. Effects of $\mathrm{HA}$ and NA glycosylation pattern changes on the transmission of avian influenza A(H7N9) virus in guinea pigs. Biochem. Biophys. Res. Commun. 2016, 479, 192-197. [CrossRef] [PubMed]

36. Reynolds, J.; Torremorell, M.; Craft, M.E. Mathematical modeling of influenza A virus dynamics within swine farms and the effects of vaccination. PLoS ONE 2014, 9, e106177. [CrossRef]

37. Cador, C.; Rose, N.; Willem, L.; Andraud, M. Maternally derived immunity extends swine influenza A virus persistence within farrow-to-finisch pig farms: Insights from a stochastic event-driven metapopulation model. PLoS ONE 2016, 11, e0163672. [CrossRef]

38. Pitzer, V.E.; Aguas, R.; Riley, S.; Loeffen, W.L.A.; Wood, J.L.N.; Grenfell, B.T. High turnover drives prolonged persistence of influenza in managed pig herds. J. R. Soc. Interface 2016, 13, 20160138. [CrossRef]

39. Cador, C.; Andraud, M.; Willem, L.; Rose, N. Control of endemic swine flu persistence in farrow-to-finish pig farms: A stochastic metapopulation modeling assessment. Vet. Res. 2017, 48, 58. [CrossRef]

(C) 2020 by the authors. Licensee MDPI, Basel, Switzerland. This article is an open access article distributed under the terms and conditions of the Creative Commons Attribution (CC BY) license (http://creativecommons.org/licenses/by/4.0/). 